\title{
Familial spastic paraplegia, bilateral sensorineural deafness, and intellectual retardation associated with a progressive nephropathy
}

\author{
J S FITZSIMMONS*, A R WATSON $\dagger$, D MELLOR + , AND \\ P R GUILBERT* \\ From ${ }^{*}$ the Department of Clinical Genetics and +Department of Paediatrics, City Hospital, Nottingham.
}

SUMMARY We present a family in which at least four persons have evidence of an inherited disorder comprising a variable spastic paraplegia, bilateral sensorineural deafness, intellectual retardation, and a progressive nephropathy. Focal segmental proliferative lesions with sclerosis suggestive of mesangial IgA nephropathy (Berger's disease) were found on renal biopsy in two affected persons. The glomerular basement membrane showed none of the changes characteristic of Alport's syndrome. Males and females are affected and the segregation of the disease is consistent with dominant transmission.

Hereditary spastic paraplegia may be conveniently divided into the so called pure type ${ }^{1}$ and the syndromic group. ${ }^{2}$ In the former there are generally no abnormalities outside the spinal cord and the spasticity and weakness primarily involve the lower limbs. The condition is relatively benign, usually dominantly transmitted, and has a variable age of onset. Syndromic spastic paraplegia may show a variety of associated features including abnormalities of the skin, central nervous system, or optic nerves. The number of syndromes with spastic paraplegia as a major feature continues to increase, ${ }^{3}$ but there appears to be no previous report of its association with a hereditary nephropathy.

\section{Case reports}

\section{IV.7 (FIG 1)}

This boy, born on 13.10 .72 , was the only child of his mother's first marriage. The pregnancy was said to be uneventful and he was delivered normally, but unexpectedly, at home, with a birth weight of $1.6 \mathrm{~kg}$ and an estimated gestation of 32 weeks. His early development was said to be normal, but his speech was delayed and he had only limited vocabulary at the age of three. By the age of five he was found to have bilateral hearing loss of 60 to 90 decibels and was recommended hearing aids. His intelligence was low and he was educated at a special school from the age of five. He had been slow to walk and from the

Received for publication 22 January 1987.

Revised version accepted for publication 19 February 1987 age of four had a history of frequent falls. In 1981 aged eight, he was referred for neurological assessment because of his walking difficulties and slow general development. At that time he was found to have scissors gait and obvious spasticity in his lower limbs. Muscle tone was markedly increased particularly in the adductors of the hips and calf muscles. He had bilateral ankle clonus with increased knee and ankle jerks and extensor plantar responses. The muscle tone in his arms appeared normal but the reflexes were brisk. There were no sensory changes and no evidence of cranial nerve abnormalities. His general condition at that time was good; his height was on the 3rd centile, his weight on the 10th centile, and head circumference

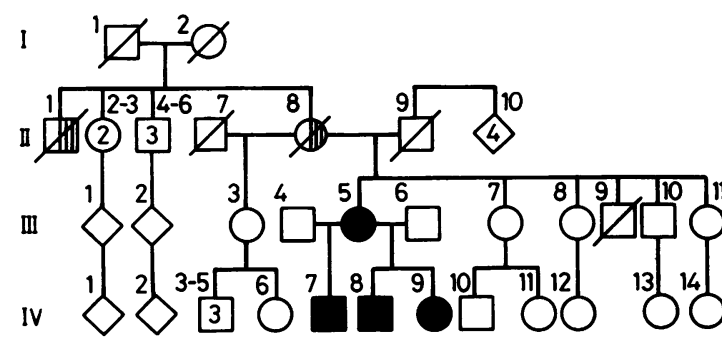

Congenital nephropathy, deafness, spastic paraplegia Renal disease (historical evidence)

FIG 1 Family pedigree. 
on the 50th centile. The only other minor abnormality noted was bilateral clinodactyly of the little fingers. In 1983, at 11 years of age, his age equivalent score on the British picture vocabulary scale was four years eight months. His walking gradually deteriorated and by 1985 he required calipers.

During neurological follow up he was found to have persistent proteinuria and was referred to a paediatric renal clinic at the age of 12 years eight months. Urine analysis showed $3 \mathrm{~g} / 1$ of protein and a trace of blood and a 24 hour urine collection showed a protein excretion of $2 \cdot 9 \mathrm{~g}$. Plasma electrolytes and blood urea were normal but the serum albumin was reduced to $28 \mathrm{~g} / 1$ (normal 30 to $50 \mathrm{~g} / 1$ ). The glomerular filtration rate was reduced at 36 $\mathrm{ml} / \mathrm{min}$ (normal range $90 \pm 15 \mathrm{ml} / \mathrm{min}$ ). Serum immunoglobulin, autoantibodies, and complement levels were all normal. A percutaneous renal biopsy in 1985 showed $50 \%$ of glomeruli to be globally sclerosed with patchy tubular atrophy. One glomerulus showed sclerosis of a pre-existing segmental proliferative lesion and others had evidence of an increase in mesangial cells and matrix with segmental accentuation (fig $2 \mathrm{a}, \mathrm{b}$ ). Immunofluorescence showed diffuse mesangial $\operatorname{IgA}, \operatorname{IgG}$, and C3 with multiple electron dense deposits in the mesangium on electron microscopy.

Since the time of diagnosis renal function has remained stable. Although free of oedema, the patient continues to have biochemical indices indicative of the nephrotic syndrome with a serum albumin of $24 \mathrm{~g} / 1$ and heavy proteinuria of 3 to 5 $\mathrm{g} / 24$ hours.

\section{5}

The mother of the proband was born on 4.8.52. At the time of referral of the proband she was already under review by the renal physicians. She was a poor historian with low intelligence, but her general health was reputed to have been good. She required special education from the age of 11 . Recurrent episodes of cystitis occurred up to the age of 14 . Proteinuria and hypertension were noted during her second pregnancy in 1977 and again in a subsequent pregnancy in 1979. She remained hypertensive during 1980 with a blood pressure of $160 / 100$ and at her request was sterilised. In 1982 she was referred to the renal clinic because of the continuing hypertension. An intravenous urogram showed a small left kidney and renal biopsy showed focal and segmental mesangial proliferative lesions with positive immunofluorescence for $\operatorname{IgA}$ and $\mathrm{C} 3$ and electron dense mesangial deposits on electron microscopy (fig 2c). The changes were similar to those noted in the proband. In 1986 her renal function deteriorated further and after six months continuous ambulatory peritoneal dialysis she underwent renal transplantation which has so far been successful.

She had bilateral hearing impairment for many years but did not know when this was first noted. She had been recommended hearing aids but generally preferred to manage without them. She admitted to difficulty with walking from the age of 28 years and on examination by a neurologist in 1984 she was found to have spastic paraplegia with evidence of spasticity in her calf muscles. All the lower limb reflexes were brisk and there was a bilateral extensor plantar response. No sensory changes were noted and no abnormalities were detected in the cranial nerves.

\section{8}

This male infant, born on 26.11 .77 , was delivered by forceps at 31 weeks' gestation with a birth weight of $1.6 \mathrm{~kg}$. His early development was delayed and he was confirmed as having bilateral hearing loss at five years and was recommended hearing aids. $\mathrm{He}$ subsequently required education at a special school. On examination by a neurologist in 1985, he was found to have lower limb hyperreflexia with spasticity. Urine examination at the same time confirmed a persistent proteinuria, but renal function tests were otherwise normal. On examination in the genetic clinic at the age of eight years, his height was $150 \mathrm{~cm}$ (25th centile) and his head circumference was on the 50th centile. He was normotensive and his general condition was good. There was no obvious facial dysmorphism, but he was noted to have a high palate. Lower limb spasticity was confirmed but the cranial nerves appeared normal. He remains under the supervision of a paediatric nephrologist and renal biopsy is not contemplated at present as the likely pathological lesions have been defined in the mother and proband.

\section{9}

This female, born on 17.8.79, was delivered by caesarean section at term with a birth weight of 1.93 $\mathrm{kg}$. In 1983 aged four she was referred to a paediatric neurologist because she was noted to drag her right leg. On examination she was found to have evidence of spastic paraplegia with increased tone in her calves, bilateral ankle clonus, and brisk knee and ankle jerks. She had a bilateral Babinski response and a tendency to walk on her tiptoes. There was no sensory loss, her upper limbs were normal, and her cranial nerves intact. At that time her urine was normal on routine testing. In 1984 she was found to have a moderate bilateral sensorineural deafness and she was fitted with hearing aids. 

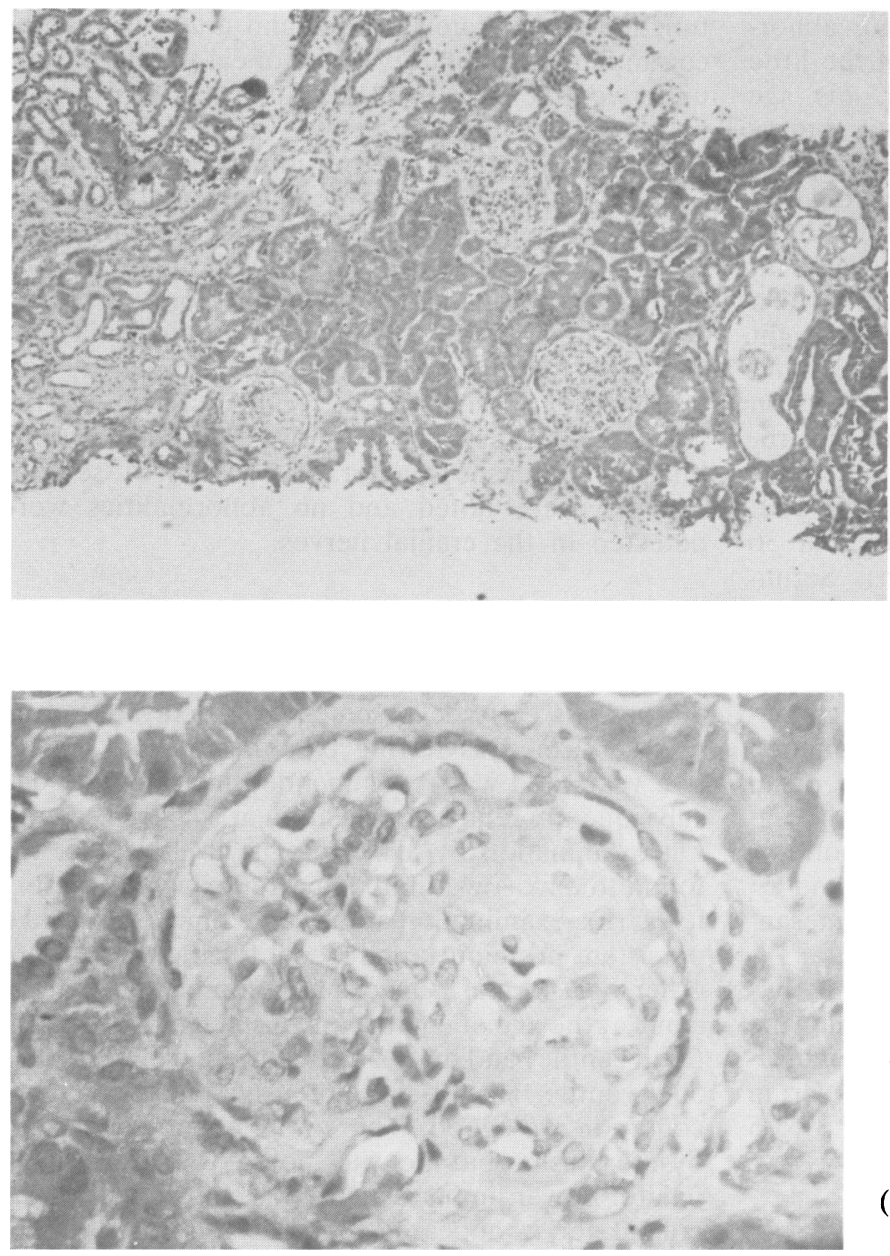

FIG 2 (a) Biopsy appearance of proband IV.7 showing patchy tubular atrophy and interstitial fibrosis. (b) One glomerulus showing sclerosis of a segmental proliferative lesion. (c) Biopsy appearance of III.5 showing hyalinised glomerulus and mild mesangial proliferation in the other.

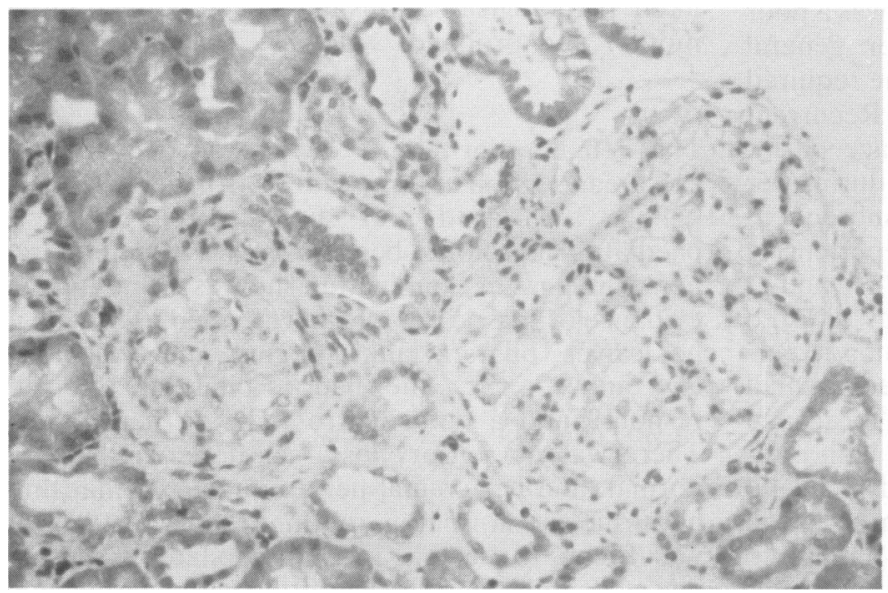


In 1985 proteinuria was found for the first time and has persisted. Her blood pressure and renal function tests remain normal but there has been an increase in her spasticity, and in 1986 she was recommended calipers. On examination in the genetic clinic at the age of six, her height was $108 \mathrm{~cm}$ (3rd to 10th centile), weight $16.4 \mathrm{~kb}$ ( $3 \mathrm{rd}$ centile), and head circumference on the 50th centile. She walked with an obvious spastic gait, but had a normal blood pressure.

\section{8}

II. 8 died at the age of 53 of a cerebral accident. Her blood pressure at the time was $150 / 80$. She had a history of renal problems and an IVP was reported as showing unilateral hydronephrosis, but she failed to attend hospital for further investigation.

\section{9}

II. 9 was reputed to have renal disease but no further details have been obtained. Other family members declined examination.

\section{Discussion}

The association of chronic hereditary renal disease and deafness is well documented. The most common disorder of this type is Alport's syndrome which shows considerable variability in clinical manifestation, natural history, and mode of inheritance. ${ }^{45}$ The histological appearances on renal biopsy in the family we describe are more typical of mesangial IgA nephropathy (Berger's disease). ${ }^{6} 7$ Although progressive nephropathy and deafness are present in our family, there were none of the characteristic glomerular basement membrane changes on electron microscopy such as thickening, focal thinning, or splitting, to suggest Alport's syndrome. To our knowledge there have been no other reports of spastic paraplegia and deafness in patients suffering from mesangial IgA nephropathy.

Mesangial IgA nephropathy has been reported in families and a large number of diseases have been reported as being associated with the condition, including liver and gastrointestinal disorders. ${ }^{6}$ Many of these associations may be a chance occurrence of IgA nephropathy with disorders in which there is an immunological disturbance. No such disturbance has been seen in our family and the immunological and serological parameters are all normal.

Kincaid-Smith and Nicholls ${ }^{6}$ have summarised the reported associations between IgA nephropathy and HLA groups. There appeared to be an initial strong association between the presence of HLA-Bw35 and a poor prognosis of $\operatorname{IgA}$ nephropathy but this has not been borne out in other reported series.
It is noteworthy that the 13 year old male proband in our report has proteinuria, impaired renal function, and advanced sclerosis on renal biopsy. These are usually regarded as late manifestations of mesangial IgA nephropathy. ${ }^{78}$ Though males are more commonly affected with this condition, they deteriorate more often and more severely than females, a situation analogous to that in Alport's syndrome.

The neurological lesions noted in all the affected subjects in this report appear to be progressive and have the characteristic features of spastic paraplegia. As anticipated, the most obvious changes are in the lower limbs with hyperreflexia and variable difficulty in walking. Sensation remains intact and there is no evidence of an associated polyneuropathy. Charcot-Marie-Tooth disease has occasionally been reported with an associated nephropathy, ${ }^{9}$ but the clinical features of this condition are significantly different from those in the patients in this report. All the affected patients have evidence of intellectual impairment which does not appear to be entirely attributable to educational problems associated with their deafness. Social factors and family circumstances may be playing a part in this family, but some impairment of intellect is not infrequently noticed in patients with this so called pure spastic paraplegia. The hearing impairment is of moderate degree and has no features distinguishing it from congenital bilateral sensorineural deafness in other syndromes.

\section{Conclusion}

There have been no previous reports of spastic paraplegia associated with an hereditary nephropathy and deafness. Dominant transmission of isolated spastic paraplegia is recognised, but the consistent segregation of this neurological lesion with the renal and auditory problems in the subjects in this family appears to confirm a syndromic association. The possibility that there are two separate genetic conditions in the family is untenable and this association has not been previously reported. The segregation of the disease is consistent with autosomal dominant transmission, but $\mathrm{X}$ linked inheritance cannot be confidently excluded on the basis of this one reported affected family.

We thank Professors R E Cotton and D R Turner for review of the histology and Dr R P Burden for clinical information. We are grateful to Miss C E Callaway for typing the manuscript.

\section{References}

1 Harding AE. Hereditary 'pure' spastic paraplegia: a clinical and genetic study of 22 families. J Neurol Neurosurg Psychiatry $1981 ; 44: 871-83$. 
2 Bundey S. Spastic paraplegia. In: Genetics and neurology. Edinburgh: Churchill Livingstone, 1985: 241-61.

${ }^{3}$ Chevenix-Trent G, Leschner R, Mamunes P. Spastic paresis, glaucoma and mental retardation-a probable autosomal recessive syndrome? Clin Genet 1986;30:416-21.

${ }^{4}$ Gubler M, Levy M, Broyer M, et al. Alport's syndrome: a report of 58 cases and a review of the literature. Am J Med 1981;70:493-505.

5 Grundfeld JP. The clinical spectrum of hereditary nephritis. Kidney Int 1985;27:83-92.

${ }^{6}$ Kincaid-Smith P, Nicholls K. Mesangial IgA nephropathy. Am J Kid Dis 1983;3:90-102.
${ }^{7}$ Nicholls KM, Fairley KF, Dowling JP, Kincaid-Smith P. The clinical course of mesangial IgA associated nephropathy in adults. $Q J$ Med 1984;54:227-50.

${ }^{8}$ Droy D. Natural history of primary glomerulonephritis with mesangial deposits of IgA. Contrib Nephrol 1976;2:150-7.

9 Lemieux G, Neemeh JA. Charcot-Marie-Tooth disease and nephritis. Can Med Assoc J 1967;97:1193-8.

Correspondence and requests for reprints to $\mathrm{Dr} J \mathrm{~S}$ Fitzsimmons, Department of Clinical Genetics, City $\vec{\circ}$ Hospital, Hucknall Road, Nottingham NG5 1PB. 